\title{
Correction to: Meta-analysis of Placebo Response in Randomized Clinical Trials of Antipsychotic Drugs Using PANSS Focusing on Different Approaches to the Handling of Missing Data
}

\author{
Akiko Matsusaki $^{1}$ (D) Masayuki Kaneko ${ }^{1} \cdot$ Mamoru Narukawa $^{1}$
}

Published online: 19 June 2018

๑) Springer International Publishing AG, part of Springer Nature 2018

\section{Correction to: Clin Drug Investig}

https://doi.org/10.1007/s40261-018-0661-1

In the original publication, the Estimate column with values under Multivariate meta-regression (LOCF) in Table 2 was missed. The corrected Table 2 is shown below:

The original article has been corrected.

The original article can be found online at https://doi.org/10.1007/ s40261-018-0661-1.

\section{Akiko Matsusaki}

matsusakiakiko_kitasato@yahoo.co.jp

1 Department of Clinical Medicine (Pharmaceutical

Medicine), Graduate School of Pharmaceutical Sciences,

Kitasato University, 5-9-1 Shirokane, Minato-ku,

Tokyo 108-8641, Japan 
Table 2 Findings by meta-regression analyses in placebo-controlled randomized clinical trials by MMRM and LOCF

\begin{tabular}{|c|c|c|c|c|c|c|}
\hline \multirow[t]{2}{*}{ Variable } & \multicolumn{3}{|c|}{ Univariate meta-regression (MMRM) } & \multicolumn{3}{|c|}{ Univariate meta-regression (LOCF) } \\
\hline & Estimate & SE & $p$ value & Estimate & SE & $p$ value \\
\hline \multicolumn{7}{|l|}{ Patient factors } \\
\hline Diagnosis & & & & -9.304 & 3.107 & 0.003 \\
\hline Severity & -0.127 & 0.104 & 0.225 & -0.101 & 0.164 & 0.539 \\
\hline Age & 0.747 & 0.480 & 0.120 & 0.707 & 0.483 & 0.144 \\
\hline$\%$ Male & 0.149 & 0.094 & 0.111 & 0.104 & 0.112 & 0.352 \\
\hline \multicolumn{7}{|l|}{ Investigator factor } \\
\hline Rater training & -4.321 & 2.032 & 0.033 & -2.384 & 2.279 & 0.295 \\
\hline \multicolumn{7}{|l|}{ Study design factors } \\
\hline Study duration & -2.130 & 0.740 & 0.004 & 2.267 & 0.757 & 0.003 \\
\hline Placebo lead-in & -1.039 & 1.878 & 0.580 & 5.618 & 2.057 & 0.006 \\
\hline Number of treatment arms & -1.241 & 1.205 & 0.303 & 0.774 & 1.123 & 0.491 \\
\hline Placebo randomization rate & -0.014 & 0.236 & 0.953 & 0.160 & 0.162 & 0.324 \\
\hline Regimen & 2.466 & 1.658 & 0.137 & -1.191 & 2.309 & 0.606 \\
\hline Active comparator & -2.324 & 1.818 & 0.201 & 4.407 & 2.573 & 0.087 \\
\hline Treatment setting & -3.463 & 1.425 & 0.015 & -1.329 & 2.737 & 0.627 \\
\hline \multicolumn{7}{|l|}{ Operational factors } \\
\hline Number of study sites & -0.096 & 0.048 & 0.047 & -0.023 & 0.049 & 0.640 \\
\hline Number of countries & -0.653 & 0.297 & 0.028 & -0.328 & 0.339 & 0.333 \\
\hline Study period & -0.042 & 0.152 & 0.781 & 0.126 & 0.144 & 0.381 \\
\hline Enrollment Speed & 5.106 & 1.674 & 0.002 & -0.782 & 3.293 & 0.812 \\
\hline \multirow[t]{2}{*}{ Variable } & \multicolumn{3}{|c|}{ Multivariate meta-regression (MMRM) } & \multicolumn{3}{|c|}{ Multivariate meta-regression (LOCF) } \\
\hline & Estimate & SE & $p$ value & Estimate & SE & $p$ value \\
\hline \multicolumn{7}{|l|}{ Patient factors } \\
\hline Diagnosis & & & & -4.490 & 3.297 & 0.173 \\
\hline \multicolumn{7}{|l|}{ Investigator factor } \\
\hline Rater training & -3.607 & 2.990 & 0.228 & & & \\
\hline \multicolumn{7}{|l|}{ Study design factors } \\
\hline Study duration & 0.866 & 1.231 & 0.482 & 1.567 & 0.747 & 0.036 \\
\hline Placebo lead-in & & & & 2.994 & 2.119 & 0.158 \\
\hline Active comparator & & & & 1.914 & 2.384 & 0.422 \\
\hline Treatment setting & -4.217 & 1.971 & 0.032 & & & \\
\hline \multicolumn{7}{|l|}{ Operational factors } \\
\hline Number of study sites & 0.149 & 0.092 & 0.106 & & & \\
\hline Number of countries & -0.864 & 0.399 & 0.030 & & & \\
\hline Enrollment speed & 3.876 & 2.943 & 0.188 & & & \\
\hline
\end{tabular}

PANSS positive and negative syndrome scale, $L O C F$ last observation carried forward, MMRM mixed-effect models for repeated measures 